\title{
The impact of the COVID-19 pandemic on renal transplantation in the UK
}

\author{
Authors: Videha Sharma, ${ }^{\mathrm{A}}$ Alex Shaw, ${ }^{\mathrm{A}}$ Marcus Lowe, ${ }^{\mathrm{B}}$ Angela Summers, ${ }^{\mathrm{C}}$ David van Dellen ${ }^{\mathrm{D}}$ and Titus Augustine ${ }^{\mathrm{E}}$
}

\begin{abstract}
COVID-19 is impacting provision of renal transplantation in the UK with a reduction in clinical activity. Publicly available Renal Registry and NHS Blood and Transplant reports were analysed to model the number of missed transplant opportunities, waiting list size and change in dialysis population over a six-month period starting 5 March 2020. An estimated 1,670 kidney transplant opportunities may be lost, which will lead to 6,317 active patients on the kidney-alone waiting list, compared to 4,649 based on usual activity estimates. This will result in 1,324 additional patients on dialysis who would otherwise have been transplanted. COVID-19 will lead to a marked loss of transplant opportunities and a significantly larger national waiting list. The existing strain on dialysis capacity will be exacerbated as patients remain on dialysis as the only available form of renal replacement therapy. These findings will help inform policy and service specific strategies.
\end{abstract}

KEYWORDS: COVID-19, kidney transplantation, NHS, renal insufficiency, renal replacement therapy

DOI: 10.7861/clinmed.2020-0183

\section{Introduction}

Kidney transplantation is the most common form of renal replacement therapy in the UK and provides a sustained cure for end-stage renal disease (ESRD). ${ }^{1}$ Transplant activity is coordinated centrally by NHS Blood and Transplant (NHSBT) and over 3,000

\footnotetext{
Authors: ${ }^{\mathrm{A}}$ clinical research fellow in transplantation, Manchester University Hospitals NHS Foundation Trust, Manchester, UK and

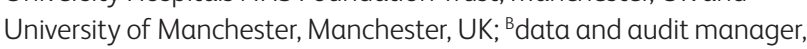
Manchester University Hospitals NHS Foundation Trust, Manchester,

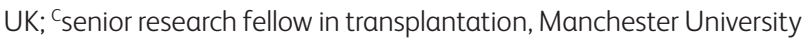
Hospitals NHS Foundation Trust, Manchester, UK and University of Manchester, Manchester, UK; ${ }^{D}$ Consultant transplant surgeon, Manchester University Hospitals NHS Foundation Trust, Manchester, UK and University of Manchester, Manchester, UK; Eprofessor of clinical transplantation and consultant transplant surgeon, Manchester University Hospitals NHS Foundation Trust, Manchester, UK and University of Manchester, Manchester, UK
}

renal transplants across 23 centres are performed each year. ${ }^{2}$ Transplantation provides significant benefits compared to dialysis and is the renal replacement therapy of choice in all eligible patients. ${ }^{3}$ In spite of increasing access to transplantation in the UK, deceased donor waiting times remain high with a median wait of 3 years, predominantly due to a paucity of suitable organ donors. ${ }^{2}$ Most patients are therefore established on dialysis for some time prior to transplantation. ${ }^{4}$ Several national strategies are in place to increase transplant rates including the use of marginal donors and the opt-out consent system. ${ }^{5}$

The global COVID-19 pandemic reached the UK on 31 January 2020, with the first recorded mortality on 5 March $2020 .{ }^{6}$ Caused by the novel coronavirus (SARS-CoV-2), it mostly presents as a respiratory illness, although $25 \%$ can have gastrointestinal symptoms. ${ }^{7}$ Disease severity can range from mild flu-like symptoms to an acute respiratory distress syndrome with the requirement for ventilatory support. ${ }^{8}$ It is highly transmissible and carries a significantly higher mortality than other coronaviruses. ${ }^{9}$ As the pandemic establishes itself in the UK, the NHS has been forced to rapidly evolve existing care models in preparation for significant front-line service pressures. In particular, NHS England mandated the suspension of all non-urgent elective surgery from 15 March 2020 to create hospital capacity for COVID-19 related activity. The majority of UK transplant centres appropriately suspended kidney transplant activity in response to this national challenge. In particular, living donor transplantation was halted at all centres and a limited number of units continue with selected deceased donor activity..$^{10}$ Decisions to continue deceased donor transplantation may be based on local severity of the pandemic as there is a geographic variation across the UK. Intensive care unit (ICU) capacity, emergency theatre access and staff resources further play a role in centres' capacity to continue to deliver services. The uncertainty in risks combined with resource and workforce challenges make decisions around transplantation highly complex at present.

The consequences of infection with novel coronavirus for renal transplant recipients remain poorly characterised. Post-transplant immunosuppressed patients are known to be susceptible to opportunistic viral infection, with coronaviruses previously reported as a causative agent..$^{11}$ The role of immunosuppression in the context of COVID-19 is still being explored and potential donor transmission rates are unestablished. Early reports suggest elective surgery on asymptomatic patients during the incubation period of COVID-19 carries a significant risk of early post-operative mortality. ${ }^{12-14}$ The overall impact for the UK is an acute reduction in 
renal transplant activity, but the effects on waiting list times and dialysis provision are unquantified. The immediate national focus is appropriately on pandemic response, but consideration should be given to planning and resource allocation for medium- and long-term effects, which include challenges for service planning and resource allocation.

This study aims to help quantify the impact of the COVID-19 pandemic on renal transplantation in the UK..$^{15}$ The primary aim is to predict the monthly number of transplants that will not be performed ('missed opportunities') due to suspension of transplant activity secondary to COVID-19 and model the size of the prospective waiting list. The secondary aim is to predict how many additional patients may require dialysis due to lack of transplantation.

\section{Methods}

The latest NHSBT and UK Renal Registry (UKRR) annual reports were included in the study. ${ }^{2,16,17}$ These national reports are collated by NHSBT and the UK Renal Association respectively. Both reports include patient-level data submitted by all 23 transplant units and 84 renal centres in the UK. NHSBT also maintains an overview of national waiting list figures and living and deceased donor transplant activity.

The day of the first reported death in the UK attributed to COVID-19 (5 March 2020) was used as 'day 0' to model the loss of transplant opportunities during the pandemic. ${ }^{6}$ The UK government has modelled a minimum of a 3 -month period of impact, but this remains an estimation. For this reason, we decided to model a period of 3-6 months. The modelling was performed on 2 April 2020.

\section{Lost transplant opportunities}

All adult and paediatric kidney transplants from 1 April 2018 to 31 March 2019 were included. This included kidney-alone transplants, and kidney transplants forming part of a multi-organ transplant such as simultaneous pancreas-kidney transplant. We utilised the reported activity of the 12-month period as stated in the latest report to model the expected activity that would have taken place normally without any pandemic effect. For purposes of this study, we worked on the assumption that transplant activity would be uniformly even across the 12 months. This provided the number of 'missed opportunities' per month, and allowed modelling of the size of the prospective transplant waiting list and dialysis population.

\section{Kidney transplant waiting list}

The kidney-alone transplant waiting list at 'day 0' was used as a starting point for modelling the size of the waiting list at 3-6 months. Over the course of the last decade the kidney transplant waiting list has been gradually declining. This is a result of increasing transplant activity, though this appears to have plateaued over the last two years. A number of patients die on the waiting list and a number are removed, typically because they become too unwell for a transplant.

\section{UK dialysis population}

The UKRR records the haemodialysis and peritoneal dialysis capacity of all UK renal centres. These were collated to provide an estimate on prevalent dialysis numbers on 'day 0', as well as new incident patients starting dialysis over the study period. These figures were used to model the size of the additional UK dialysis population as a result of curtailed transplant activity during the pandemic.

\section{Modelling analysis}

The modelling assumed that activity during the study period would be identical to the figures reported in the latest NHSBT and UKRR reports. We identified the key coefficients such as number of transplants performed, waiting list size and prevalent dialysis numbers and modelled these with the variable of number of months since 'day 0'. Modelling was performed on Microsoft Excel. ${ }^{\circledR}$ Detailed formulae are available in the supplementary data.

\section{Ethical approval}

This study analysed data from publicly available NHSBT and UKRR reports. No explicit consent to use this data was deemed necessary as they are publicly available and the reports were cited accurately. Since the data in the reports are fully anonymised, no ethical permission was required.

\section{Results}

We included data on 3,597 kidney transplants in the 2018/2019 NHSBT annual report over a 12-month period. ${ }^{2,16}$ There were 1,020 living donor kidney transplants and 2,577 deceased donor transplants. The overall active kidney transplant waiting list at the end of the financial year (31 March 2019) was 4,954 patients, which was a $2 \%$ decrease from the previous year.

\section{Lost transplant opportunities}

There was a mean of 300 kidney transplants each month between 1 April 2018 and 31 March 2019 (85 living donor and 215 deceased donor transplants). This indicates that routine activity over a 3-month period would predict 900 kidney transplants. Between 'day 0' and the time of the analysis, 128 kidney transplants were performed. The COVID-19 pandemic is thus expected to lead to a potential loss of up to 1,672 transplant opportunities between 5 March 2020 and the 5 September 2020 (6 months). Table 1 shows the number of transplants projected based on the anticipated activity in normal and pandemic circumstances, as well as the number of lost opportunities.

\section{Kidney transplant waiting list}

There were 4,748 patients active on the list on 5 March 2020 ('day 0 '). The modelling has shown that between 772 and 1,672 lost opportunities will result in a $16.3 \%-35.2 \%$ increase in waiting list size (Fig 1). Projecting the trend of a $4.2 \%$ reduction between 31 March 2019 and 5 March 2020 ('day 0'), the size of the list would have normally decreased by $1-2 \%$ over the same period. If activity continued as normal, the list would have expected to reduce by 17 patients a month; however, the modelling predicts a relative increase of up to 283 patients per month.

\section{UK dialysis population}

Many transplant opportunities missed during the COVID-19 pandemic would have benefitted patients currently on dialysis. 
Table 1. Expected cumulative number of transplants performed in normal times and during the COVID-19 pandemic

\begin{tabular}{llllll} 
& $\mathbf{3}$ months & $\mathbf{4}$ months & $\mathbf{5}$ months & $\mathbf{6}$ months & Lost opportunities \\
Normal activity & 900 & 1200 & 1500 & 1800 & $\mathrm{n} / \mathrm{a}$ \\
COVID-19 pandemic $^{*}$ & 128 & 128 & 128 & 128 & $772-1672$ \\
\hline
\end{tabular}

*Only includes transplants performed from 5 March 2020 until time of analysis (2 April 2020).

$62 \%$ of living donor transplants go to dialysis patients and a 3-6-month suspension of the living donor programme would lead to 159-317 additional dialysis patients. The reduction of the deceased donor activity has even greater impact, as $87 \%$ of deceased donor transplants are for established dialysis patients. With nil deceased donor transplantation, a further 1,121 patients may be on dialysis who would have been transplanted under normal circumstances. Table 2 shows the number of additional dialysis patients there will be, based on the projected number of missed transplant opportunities.

The reduction in transplant activity will result in a total of 720-1,438 patients continuing to require dialysis who would otherwise have been transplanted. The UKRR states there were 29,064 dialysis patients in the UK on 31 December 2017, and the number had been increasing by $2.0 \%$ per year over the previous two years. Assuming this trend continued until 'day 0', there would have been approximately 30,337 dialysis patients in the UK. The figures from our modelling would thus increase the dialysis population in addition to normal growth by a further $4.7 \%$ over 6 months.

\section{Discussion}

This study has quantified the impact of the COVID-19 pandemic on renal transplant activity in the UK. The modelled loss of transplant opportunities will result in substantially increased waiting list numbers and times. This will impact dialysis capacity

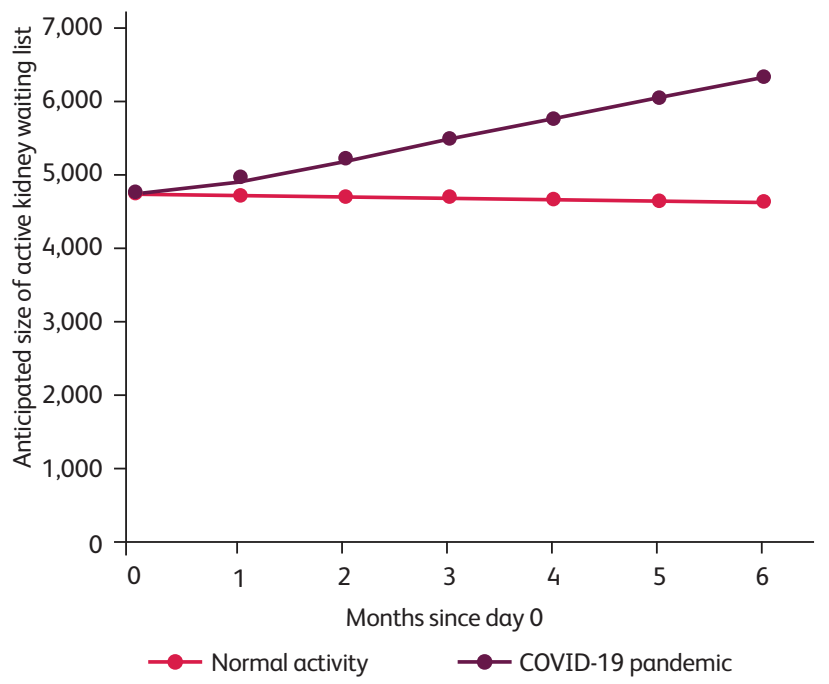

Fig 1. Predicted change in the size of the kidney-alone waiting list during the COVID-19 pandemic.
Table 2. Additional dialysis patients in the UK over

\section{the next 3-6 months}

\begin{tabular}{|c|c|c|c|}
\hline \multirow[t]{2}{*}{$\begin{array}{l}\text { Months after } \\
\text { 'day 0' }\end{array}$} & \multicolumn{3}{|c|}{$\begin{array}{l}\text { Additional dialysis patients due to missed } \\
\text { transplants from: }\end{array}$} \\
\hline & $\begin{array}{l}\text { Deceased } \\
\text { donors }\end{array}$ & $\begin{array}{l}\text { Living } \\
\text { donors }\end{array}$ & Total \\
\hline 3 & 561 & 159 & 720 \\
\hline 4 & 748 & 211 & 959 \\
\hline 5 & 935 & 264 & 1199 \\
\hline 6 & 1,121 & 317 & 1,438 \\
\hline
\end{tabular}

"This has not been adjusted for the 128 transplants recorded from 'day 0 ' till time of analysis (2 April 2020).

and provision, with additional inevitable morbidity and mortality. This study demonstrates the value of publicly available UK-based national data, allowing rapid analysis, predict service impact and inform urgent strategic policy-making.

\section{Transplantation during the COVID-19 pandemic}

There has been no national policy to halt organ donation and transplantation across the UK. At the time of analysis, however, national activity has come to a near stand-still..$^{10}$ This study shows that from 5 March 2020 onwards, between 772 and 1672 transplant opportunities will be missed over 3-6 months. This will greatly impact patients and families. Concerted efforts must be made to provide communication and support during the pandemic. Using digital technology and telemedicine is proving crucial in maintaining patient contact and health service delivery. ${ }^{18}$ Transplant patients have been shown to have favourable attitudes towards using health information technology and COVID-19 may be a stimulus to drive change in the way services are delivered..$^{19,20}$

Transplantation provides superior outcomes and quality of life parameters for ESRD; however, the adjuvant immunosuppression required during and after the surgical procedure may expose recipients to additional risk. ${ }^{21,22}$ Recent case series from the UK and Italy have reported a mortality of $7-25 \%$ of COVID-19 in posttransplant patients, adding to the concern that these patients suffer significantly adverse outcomes. ${ }^{23,24} \mathrm{~A}$ post-mortem analysis of native kidneys from six patients with confirmed COVID-19 demonstrated a high tropism of SARS-CoV-2 for the human kidney. ${ }^{25}$ Across Europe, many national bodies continue to support transplantation but stress the importance of donor COVID-19 
testing. ${ }^{26,27}$ This is advocated to reduce the risk of transmission to recipients, but also to protect organ retrieval teams. The primary challenge appears to be that a clear risk/benefit assessment of transplantation during the active COVID-19 pandemic is still unclear. Guidance published thus far provides limited objective data for detailed risk/benefit discussions with patients. ${ }^{10,28}$ While the current onus is on individual centres to formulate local practice, national strategies are mandated to provide clear guidance to both maintain activity but more importantly provide quantifiable risk stratification. This requires a focus on understanding the consequences of COVID-19 in the context of immunosuppression, and establishing safe approaches to donation. It is imperative that the transplant community shares knowledge and experience rapidly to drive research and form a readily accessible evidence base.

\section{Post-pandemic transplant activity}

Following COVID-19, service review with cogent planning and updated resource allocation will be necessary to minimise the effects of lost activity on individual patients. While patients are not currently being added to the transplant waiting list, the rate of patients developing ESRD will be the same or even higher as a result of COVID-19. Therefore, it is envisaged that a backlog of patients will be added post-pandemic, resulting in an acute rise in the waiting list size. This study has shown the transplant waiting list may increase by $720-1,438$ active dialysis patients over 3-6 months. Dialysis significantly impacts health, with an increased risk for cardiovascular events and sepsis. ${ }^{29}$ There is emerging evidence that kidney disease is associated with higher in-hospital mortality secondary to COVID-19. ${ }^{30}$ It is postulated that the COVID-19 pandemic may disproportionally affect patients on dialysis, resulting in a reduced transplant waiting list. Under normal circumstances 22 patients die each month on the kidney-alone waiting list, and prospective studies determining the additional mortality among wait-listed patients will help characterise the impact more fully.

Halting kidney transplantation will affect living and deceased donation equally. However, deceased donor transplants will be missed opportunities due to the temporal nature of organ offers. Living donor kidney transplantation is a semi-elective procedure and may be recommenced when deemed safe. This study has shown that 255-510 living donor pairs will have been postponed by the end of 3-6 months. It will require significant resources and efforts to clear this backlog, including operating theatre capacity and anaesthetic and intensive care support. The wellbeing of potential living donors is paramount and evidencebased recommendations will be required for counselling and decision-making. Early post-transplant patients are regularly reviewed in the outpatient clinic. In the interest of minimising exposure and reducing the risk of contracting COVID-19, changes in ways of working will have to be introduced, with increased remote monitoring and local blood investigations. This envisaged increased and altered working will require rationalisation along with the several other services where activity is currently halted. Healthcare workers normally involved in transplantation are being redeployed to work in emergency departments or intensive care units, which has further contributed to cessation in transplant activity. To cope with the significant increase in workload, this trend may need to reversed with additional infrastructural support post-pandemic.

\section{Impact on workforce and resource allocation}

UK kidney transplant services function under considerable pressure during 'normal' circumstances, with periodic surges in activity. The service is delivered by highly specialised multi-disciplinary staff including surgeons, nephrologists, specialist nurses and coordinators. They provide a round-the-clock national organ retrieval and implantation service of organs from deceased donors along with elective living donation. While delivering transplant care is highly rewarding, the frequency of on-calls and high volume of out-ofhours work makes recruitment and retention challenging. This study has quantified the considerable rise in workload for transplant staff post-pandemic. Reverting back to 'normal' work practices is unlikely to be a safe and effective strategy for delivering the service. This study further shows a potential unaccounted rise in the UK dialysis population, which will require additional service provision and resources. The projected increase in demand on dialysis will further stretch a recovering NHS. Per patient costs for dialysis are over $£ 24,000$ per year, and a potential increase in the dialysis population modelled in this study would incur an additional cost of $£ 13.4$ to $£ 31.8$ million. ${ }^{31}$ This is caveated by the aforementioned, as yet unknown, additional mortality risk from COVID-19 for patients on the transplant waiting list.

\section{Limitations of this study}

Due to the novelty and unprecedented impact of the of the COVID-19 pandemic, there are no other studies to act as comparators in terms of health care provision modelling. Though the methodology for modelling this is reproducible, it was dependent on the availability of national data, which may not be the case globally. We have based our modelling on the assumption that activity during the pandemic would have been similar to the last financial year. Though activity has been near-identical between the last two years, the change in the organ donation law coming in to place in May 2020 may have positively impacted on deceased donor transplantation. ${ }^{2,5}$ The missed transplant opportunities predicted in this study may therefore underestimate the projected shortfall in activity. On an international scale, however, these databases are among the largest and most accurate sources for transplant-related population level data, which would add to the validity of our findings. In 2018, Lowe et al showed a seasonal variation in kidney transplant activity by analysing 10 years of NHSBT data. ${ }^{32}$ We have not included this variation in our modelling at present. The impact of loss of activity, however, is nonetheless expected to be significant, especially if the UK kidney transplant programme is halted entirely. Additional mortality on the waiting list and the number of patients becoming too unwell to remain on the list due to COVID-19 is unknown. This would influence our overall modelling and impact the size of the waiting list as well as dialysis capacity further. We chose the 5 March 2020 as a defining point of a change in national, regional and local strategy to shift priorities in NHS services towards preventing and treating COVID-19. Retrospectively, it may be argued that a different 'day 0' represents this change more accurately; however, given the limitations and unpredictability of the situation this was considered a fair representation.

\section{Conclusion}

Kidney transplantation provides a life-enhancing procedure for patients with ESRD and is a flagship service of the NHS. While 
the unequivocal focus is battling COVID-19 and maintaining vital frontline services, kidney transplantation will be adversely affected. This will result in ancillary pressures on national dialysis capacity and the transplant waiting list. We have quantified this impact and provided evidence for health providers to recognise this upcoming change in landscape. It is critical that kidney transplant services develop innovative solutions and expedite research to deliver transplantation as soon as possible.

\section{Supplementary material}

Additional supplementary material may be found in the online version of this article at www.rcpjournals.org/clinmedicine: S1 - Formulae, coefficients and variables used in our modelling.

\section{Acknowledgements}

The data reported here have been supplied by the UK Renal Registry of The Renal Association. The interpretation and reporting of these data are the responsibility of the authors and in no way should be seen as an official policy or interpretation of the UKRR or The Renal Association.

\section{References}

1 Schnuelle P, Lorenz D, Trede M, Van Der Woude FJ. Impact of renal cadaveric transplantation on survival in end-stage renal failure: evidence for reduced mortality risk compared with hemodialysis during long-term follow-up. J Am Soc Nephrol 1998;9:2135-41.

2 NHS Blood and Transplant. Organ donation and transplantation: activity report 2018/19. NHSBT, 2020. www.organdonation.nhs.uk/ helping-you-to-decide/about-organ-donation/statistics-aboutorgan-donation/transplant-activity-report/.

3 Oniscu GC, Brown H, Forsythe JLR. Impact of cadaveric renal transplantation on survival in patients listed for transplantation. J Am Soc Nephrol 2005;16:1859-65.

4 Meier-Kriesche HU, Kaplan B. Waiting time on dialysis as the strongest modifiable risk factor for renal transplant outcomes: a paired donor kidney analysis. Transplantation 2002;74:1377-81.

5 Department of Health Social Care. Opt-out organ donation: Max and Keira's Bill passed into law. DHSC, 2019. www.gov.uk/government/ news/opt-out-organ-donation-max-and-keira-s-bill-passed-into-law.

6 Marsh S. Coronavirus: first UK death confirmed as cases surge to 116. The Guardian, 6 March 2020.

7 Sohrabi C, Alsafi Z, O'Neill N et al. World Health Organization declares global emergency: A review of the 2019 novel coronavirus (COVID-19). Int J Surg 2020;76:71-6.

8 Zhou F, Yu T, Du R et al. Clinical course and risk factors for mortality of adult inpatients with COVID-19 in Wuhan, China: a retrospective cohort study. Lancet 2020;395:1054-62.

9 Yang $X, Y u Y, X u$ J et al. Clinical course and outcomes of critically ill patients with SARS-CoV-2 pneumonia in Wuhan, China: a singlecentered, retrospective, observational study. Lancet Respir Med 2020;8:475-81.

10 NHS Blood and Transplant. COVID-19 Bulletin Number 5. NHSBT, 2020. https://nhsbtdbe.blob.core.windows.net/umbraco-assets-corp/ 18190/covid-19-bulletin-5-02042020.pdf.

11 Kotton CN, Fishman JA. Viral infection in the renal transplant recipient. J Am Soc Nephrol 2005;16:1758-74.

12 Lei S, Jiang F, Su W et al. Clinical characteristics and outcomes of patients undergoing surgeries during the incubation period of COVID-19 infection. EClinicalMedicine 2020;21:100331.

13 Aminian A, Safari S, Razeghian-Jahromi A, Ghorbani M, Delaney CP. COVID-19 outbreak and surgical practice: unexpected fatality in perioperative period. Ann Surg 2020, in press (doi: 10.1097/ SLA.0000000000003925).
14 Li Y-K, Peng S, Li L-Q et al. Clinical and transmission characteristics of Covid-19 - a retrospective study of 25 cases from a single thoracic surgery department. Curr Med Sci 2020;40:295-300.

15 Kumar D, Manuel O, Natori Y et al. COVID-19: A global transplant perspective on successfully navigating a pandemic. Am J Transplant 2020, in press (doi: 10.1111/ajt.15876).

16 NHS Blood and Transplant. Annual report on kidney transplantation: report for 2018/2019 (1 April 2009 - 31 March 2019). NHSBT, 2019.

17 UK Renal Registry. UK Renal Registry 21st Annual Report - data to 31/12/2017. Renal Association, 2019. www.renalreg.org/reports/ data_to_end_2017/.

18 Hollander JE, Carr BG. Virtually perfect? Telemedicine for Covid-19. N Engl J Med 2020;382:1679-81.

19 Browning RB, McGillicuddy JW, Treiber FA, Taber DJ. Kidney transplant recipients' attitudes about using mobile health technology for managing and monitoring medication therapy. J Am Pharm Assoc 2016;56:450-4.

20 Zanetti-Yabur A, Rizzo A, Hayde $\mathrm{N}$ et al. Exploring the usage of a mobile phone application in transplanted patients to encourage medication compliance and education. Am J Surg 2017:214:743-7.

21 Laupacis A, Keown P, Pus N et al. A study of the quality of life and cost-utility of renal transplantation. Kidney Int 1996;50:235-42.

22 Mehta P, McAuley DF, Brown M et al. COVID-19: consider cytokine storm syndromes and immunosuppression. Lancet 2020;395:1033-4

23 Alberici F, Delbarba E, Manenti C et al. A single center observational study of the clinical characteristics and short-term outcome of 20 kidney transplant patients admitted for SARS-CoV2 pneumonia. Kidney Int 2020;pii:S0085-2538(20)30365-3.

24 Banerjee D, Popoola J, Shah S et al. COVID-19 infection in kidney transplant recipients. Kidney Int 2020, in press (doi: 10.1016/j. kint.2020.03.018).

25 Diao B, Feng Z, Wang C et al. Human kidney is a target for novel severe acute respiratory syndrome coronavirus 2 (SARS-CoV-2) infection. medRxiv 2020.03.04.20031120 (doi: 10.1101/2020.03.04.20031120).

26 Ahn C, Amer H, Anglicheau D et al. Global Transplantation COVID Report, March 2020. Transplantation 2020, in press (doi: 10.1097/ TP.0000000000003258).

27 NHS Blood and Transplant. Covid-19 donation - a summary of international correspondence. NHSBT, 2020. https://nhsbtdbe.blob. core.windows.net/umbraco-assets-corp/18016/donationinternational-reflections-17032020.pdf.

28 American Society of Transplantation. COVID-19 (Coronavirus): FAQs for organ transplantation. AST, 2020.

29 Bray BD, Boyd J, Daly C et al. How safe is renal replacement therapy? A national study of mortality and adverse events contributing to the death of renal replacement therapy recipients. Nephrol Dial Transplant 2014;29:681-7.

30 Cheng Y, Luo R, Wang K et al. Kidney disease is associated with in-hospital death of patients with COVID-19. Kidney Int 2020;97:829-38.

31 Kerr M, Bray B, Medcalf J, O'Donoghue DJ, Matthews B. Estimating the financial cost of chronic kidney disease to the NHS in England. Nephrol Dial Transplant 2012;27(suppl_3):iii73-iii80.

32 Lowe M, Maidstone R, Poulton K et al. Monthly variance in UK renal transplantation activity: a national retrospective cohort study. BM] Open 2019;9:e028786.

Address for correspondence: Mr Videha Sharma, Department of Renal and Pancreatic Transplantation, Manchester University NHS Foundation Trust, Oxford Road, Manchester M13 9WL, UK.

Email: videha.sharma@mft.nhs.uk 